\title{
Aptidão física funcional e nível de atenção em idosas praticantes de exercício físico
}

\author{
Functional physical fitness and level of attention in elderly practitioners of exercise
}

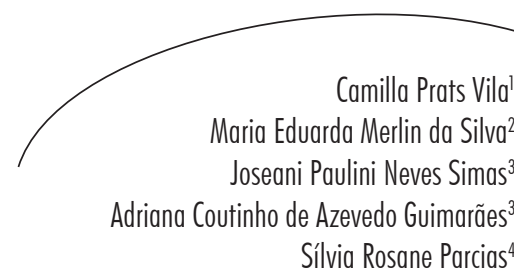

Resumo

Introdução: O envelhecimento conduz à perda das aptidões funcionais e do funcionamento cognitivo, aumentando o risco do sedentarismo. O exercício físico combate o sedentarismo e contribui para a manutenção da aptidão física e do nível de atenção no idoso. Objetivo: Analisar a aptidão física funcional e a atenção, entre dois grupos de idosas praticantes de exercício físico e sedentárias. Métodos: Amostra composta por 25 idosas, divididas em dois grupos: grupo A (12 fisicamente ativas) e grupo S (13 sedentárias). Para a coleta de dados, nos dois grupos foram utilizados: o questionário de Baecke, para selecionar as idosas; a bateria Senior Fitness Test (SFT), para avaliar a aptidão física funcional; e o Teste de Traços (TMT A e B), para avaliar a atenção. Para a análise dos dados, foram usadas as técnicas de estatística descritiva e inferencial. Resultados: No questionário de Baecke, o grupo A obteve melhor resposta que o grupo S. Os dois grupos apresentaram valores iguais nos parâmetros da aptidão física, quando comparados com os valores-padrão de referência, exceto no teste de flexibilidade dos membros superiores e de resistência aeróbica, onde apresentaram desempenho inferior. Nos testes de flexibilidade de membros inferiores; mobilidade física - velocidade, agilidade e equilíbrio; e resistência aeróbica, observou-se diferença estatisticamente significativa. No TMT, foram encontrados valores acima dos de referência para a parte $\mathrm{A}$ nos dois grupos e para a parte B apenas no grupo A, o que demonstra baixo desempenho. Conclusão: As idosas deste estudo apresentaram nível inferior nos parâmetros de flexibilidade de membros superiores e resistência aeróbica. As ativas apresentaram melhor flexibilidade de membros inferiores, melhor mobilidade
Palavras-chave: Idoso. Aptidão. Atividade Motora. Atenção.

Curso de Fisioterapia, Centro de Ciências da Saúde e do Esporte. Universidade do Estado de Santa Catarina. Florianópolis, SC, Brasil.

2 Programa de Pós-graduação em Enfermagem, Centro de Ciências da Saúde. Universidade Federal de Santa Catarina. Florianópolis, SC, Brasil.

3 Departamento de Educação Física, Centro de Ciências da Saúde e do Esporte. Universidade do Estado de Santa Catarina. Florianópolis, SC, Brasil.

4 Departamento de Ciências da Saúde, Centro de Ciências da Saúde e do Esporte. Universidade do Estado de Santa Catarina. Florianópolis, SC, Brasil. 
física (velocidade, agilidade e equilíbrio) e mais resistência aeróbica que as sedentárias. Constatou-se prejuízo na atenção e rapidez visomotora nas idosas. Enfatiza-se a importância de se praticar exercícios que estimulem as capacidades intelectuais, além das capacidades físicas dos idosos.

Abstract

Introduction: Aging leads to loss of functional abilities and cognitive functioning, increasing the risk of sedentary lifestyle. Physical exercise fights sedentary lifestyle and contributes to the maintenance of physical fitness and the level of care in the elderly. Objective: To analyze the functional physical fitness and attention, between two groups of elderly, practitioners of physical exercise and sedentary. Methods: A sample of 25 elderly women was divided into two groups: group A (12 physically active) and group S (13 sedentary). For data collection in the two groups were used: Baecke questionnaire to select the elderly, battery Senior Fitness Test (SFT) to assess functional physical fitness and Trail Making Test (TMT A and B) to assess attention. It was used descriptive and inferential statistical techniques for data analysis. Results: In the Baecke questionnaire, group A showed better response than group S. Both groups had equal values in the parameters of physical fitness, when compared with values benchmarks, except in upper limb flexibility tests and aerobics resistance, which showed lower performance. In lower limb flexibility tests; physical mobility - speed, agility, balance and aerobics resistance, there was statistically significant difference. In TMT were found values above those considered as reference in both groups and, for part B, just at the group A, which shows low performance. Conclusion: The elderly women in this study had lower level of flexibility in the parameters of the upper limbs and aerobic endurance. The physically active women had better flexibility in the lower limbs, better physical mobility (speed, agility and balance) and more aerobic endurance than sedentary ones. We found deficits in attention and visual-motor speed. It is emphasized the importance of practicing exercises that stimulate intellectual abilities, beyond the physical capabilities of elderly.

Key words: Elderly. Aptitude. Motor Activity. Attention.

\section{INTRODUÇÃO}

Aos poucos, a sociedade como um todo e sobretudo a população idosa começam a ver a atividade física como forma de prevenção e reabilitação da saúde. ${ }^{1,3} \mathrm{O}$ fortalecimento dos elementos da aptidão física (força, mobilidade e flexibilidade, entre outros) é de extrema importância para o idoso, pois tais aptidões estão diretamente associadas à independência e à autonomia do idoso, principalmente na execução de suas atividades da vida diária., ${ }^{4,5}$

O envelhecimento conduz a uma perda progressiva das aptidões funcionais do organismo, aumentando o risco de sedentarismo. Essas alterações colocam em risco a qualidade de vida do idoso, por limitar sua capacidade para realizar as atividades do cotidiano com vigor e tornar sua saúde mais vulnerável. O exercício físico, além de combater o sedentarismo, contribui de maneira significativa para a manutenção da aptidão física do idoso, seja na sua vertente da saúde como nas capacidades funcionais. Quando praticados regularmente, retardam a degeneração natural dos músculos, tendões, ligamentos, ossos e articulações, além de proporcionar músculos mais fortes, articulações flexíveis e manter o equilíbrio e a coordenação, permitindo maior mobilidade e independência.,

Odeclínio do funcionamento cognitivo ocorre como um aspecto normal do envelhecimento. ${ }^{8-11}$ As perdas no domínio cognitivo, dentre elas a atenção, contribuem para a maior redução da independência do idoso, limitando suas 
possibilidades de viver de forma satisfatória e confortável, além de restringirem sua atuação na sociedade. ${ }^{10,11}$ Para se ter sucesso na realização de uma tarefa motora difícil, é necessário haver níveis elevados de atenção, por isso a importância da atenção no comportamento motor. ${ }^{8}$

O exercício físico promove benefícios das funções cerebrais, aumentando o funcionamento e o metabolismo neuronal, melhorando a cognição. ${ }^{9-11} \mathrm{O}$ exercício praticado pelo idoso deve ter como objetivo a manutenção das capacidades intelectuais, além das capacidades físicas. ${ }^{12}$

Os benefícios da prática de atividade física são reconhecidos e amplamente divulgados, porém são poucos os idosos que realizam esta atividade de forma regular. ${ }^{13}$ Sendo assim, a forma como cada indivíduo se adapta às modificações físicas, intelectuais e sociais decorrentes da idade determinará um envelhecimento saudável ou repleto de dificuldades. ${ }^{14}$

Este estudo buscou analisar a aptidão física funcional e o nível de atenção, entre dois grupos de idosas, praticantes de exercício físico e sedentárias.

\section{METODOLOGIA}

Estudo observacional analítico do tipo transversal, realizado no período de abril a junho de 2011, analisou a aptidão física funcional e os níveis de atenção em idosas de dois diferentes grupos: $\mathrm{A}=$ praticantes de exercício físico e $\mathrm{S}=$ sedentárias.

A amostra selecionada foi composta por 25 idosas, com idade variando de 60 a 90 anos, das quais 12 eram do grupo $\mathrm{A}$, com média de idade de 68 anos $( \pm 3,2)$; e 13 do grupo S, com média de idade de 71,4 anos $( \pm 8,5)$. Foram recrutadas no Centro de Atenção à Terceira Idade (CATI) e na comunidade da grande Florianópolis, selecionadas de forma intencional.

Os indivíduos foram avaliados no CATI, em São José, que conta com atividades voltadas a idosos residentes nesse município, e nas dependências do Centro de Ciências da Saúde e do Esporte (CEFID) da Universidade do Estado de Santa Catarina (UDESC), em Coqueiros, Florianópolis, que possui um Núcleo de Atividade Física, Saúde e Gerontomotricidade (NAFISGE).

Foram adotados como critérios de inclusão no estudo: ter idade igual ou maior que 60 anos, ser do gênero feminino e ser alfabetizada. Para o grupo A, realizar exercício físico duas vezes por semana, com duração média de uma hora, há pelo menos dois anos; e apresentar escore no questionário Baecke modificado maior que 9,4. Para o grupo S, apresentar escore no questionário Baecke modificado menor que 9,4, o que confirmou o sedentarismo das participantes.

Realizou-se inicialmente uma entrevista sociodemográfica e clínica, empregada com o intuito de caracterizar a amostra, contendo os seguintes itens: idade, estado civil, escolaridade, renda familiar, ocupação e utilização atual de medicamentos. Em seguida, para assegurar que o grupo A foi composto por idosas ativas e o grupo $\mathrm{S}$ por idosas sedentárias, foi aplicado o questionário de Baecke, ${ }^{15}$ o qual investigou atividades físicas habituais realizadas nos últimos 12 meses, abrangendo as atividades físicas ocupacionais, os exercícios físicos praticados durante o tempo de lazer e as atividades físicas de locomoção. Este apresenta questões relativas ao tipo de atividades praticadas, horas por semana e quantidade de meses do ano em que essas atividades são geralmente realizadas. ${ }^{14,16}$ Uma pontuação menor que 9,4 classifica o indivíduo como inativo. ${ }^{16}$

A bateria de testes utilizada no presente estudo para avaliar a aptidão física foi a Senior Fitness Test, desenvolvida para avaliar os principais parâmetros físicos associados à capacidade funcional de adultos idosos independentes, de 60 até 90 ou mais anos de idade, como força e resistência de MMII (membros inferiores) levantar e sentar na cadeira; força e resistência de MMSS (membros superiores) - flexão do 
antebraço; flexão de MI (membros inferiores) - sentado, alcançar os membros inferiores com as mãos; mobilidade física - velocidade, agilidade e equilíbrio - levanta, caminha $2,44 \mathrm{~m}$ e volta a sentar); flexibilidade de MS (membros superiores) - alcançar atrás das costas com as mãos e resistência aeróbica (andar seis minutos). ${ }^{17}$

Para avaliação da atenção, foi utilizada a bateria neuropsicológica Teste de Traços (Trail Making Test - TMT), a qual faz parte da bateria do Consortium to Establish a Registry for Alsheimer's Disease (CERAD), adaptada para a população brasileira por Bertolucci et al. ${ }^{18}$ Esse teste avalia, além da atenção, a flexibilidade mental, a velocidade de processamento visual e função motora. Constituído de uma parte A e uma segunda parte $\mathrm{B}$ de maior complexidade, foi aplicado no tempo de 5 a 10 minutos. A parte A (TMT A) avalia a rapidez visomotora e a atenção; e a parte $\mathrm{B}$, além de avaliar a rapidez visomotora e a atenção, requer uma habilidade para mudar de estratégias e, assim, avalia a função executiva. O critério de avaliação é o tempo de execução em segundos, definindo um pior desempenho à proporção que o tempo utilizado para concluir a tarefa é maior. ${ }^{19}$

No TMT, os valores de referência por faixa etária utilizados seguiram os dados normativos propostos por Tombaugh. ${ }^{20}$ Indivíduos com tempo de execução do teste acima do valor de referência são classificados como abaixo do padrão (déficit de atenção) e os com tempo igual ou abaixo do valor de referência são classificados com nível de atenção normal e acima do padrão, respectivamente.

Os dados foram analisados no pacote estatístico SPSS - 17.0, por meio da estatística descritiva, a partir da distribuição da frequência média, desvio-padrão, variância, valor mínimo e máximo. Para verificar a normalidade da distribuição amostral, utilizou-se o teste Shapiro-Wilk; na associação das variáveis sociodemográficas intergrupos, utilizou-se o teste qui-quadrado; e na comparação intergrupos das variáveis da aptidão física e funcional e das variáveis do nível de atenção, efetuou-se o teste- $t$ para amostras independentes, adotando-se como nível de significância $\mathrm{p}<0,05$.

O estudo foi aprovado pelo Comitê de Ética em Pesquisa envolvendo Seres Humanos da UDESC, sob protocolo número 169/2010. Todos os participantes foram esclarecidos acerca dos procedimentos e objetivos do estudo e assinaram o Termo de Consentimento Livre e Esclarecido.

\section{RESULTADOS}

$\mathrm{Na}$ análise das características sociodemográficas e clínicas, não foram encontradas diferenças estatisticamente significativas entre os grupos, como representado na tabela 1. 
Tabela 1 - Caracterização da amostra entre os grupos de idosas ativas e sedentárias. São José-SC, 2011.

\begin{tabular}{|c|c|c|c|}
\hline & Grupo A (n=12) - \% & Grupo S (n=13) - \% & Valor de $\mathrm{p}$ \\
\hline Escolaridade & & & 0,124 \\
\hline Nenhuma & 25 & 7,7 & \\
\hline $1^{\circ}$ grau incompleto & 0 & 7,7 & \\
\hline $1^{\circ}$ grau completo & 25 & 15,3 & \\
\hline $2^{\circ}$ grau incompleto & 0 & 30,8 & \\
\hline Superior & 16,6 & 30,8 & \\
\hline Pós-graduação & 33,4 & 7,7 & \\
\hline Estado conjugal & & & 0,961 \\
\hline Solteira & 8,3 & 15,4 & \\
\hline Casada & 25 & 23,1 & \\
\hline Divorciada & 25 & 23,1 & \\
\hline Viúva & 41,7 & 38,4 & \\
\hline Renda mensal & & & 0,483 \\
\hline 1 a 2 salários mínimos & 58,4 & 53,8 & \\
\hline 3 a 4 salários mínimos & 0 & 7,7 & \\
\hline 5 a 6 salários mínimos & 0 & 0 & \\
\hline 7 a 8 salários mínimos & 25 & 38,5 & \\
\hline 9 salários mínimos & 8,3 & 0 & \\
\hline Mais de 9 salários mínimos & 8,3 & 0 & \\
\hline Ocupação & & & 0,554 \\
\hline Aposentada & 75,1 & 61,5 & \\
\hline Do lar & 8,3 & 0 & \\
\hline Pensionista & 0 & 7,7 & \\
\hline Aposentada e pensionista & 8,3 & 7,7 & \\
\hline Do lar e pensionista & 8,3 & 23,1 & \\
\hline Medicação & & & 0,570 \\
\hline Cardiorrespiratória & 8,3 & 7,7 & \\
\hline Endócrino & 8,3 & 0 & \\
\hline Combo & 83,4 & 84,6 & \\
\hline Sem uso & 0 & 7,7 & \\
\hline
\end{tabular}

Combo = combinação de mais de uma medicação; teste do qui-quadrado. 
Em relação ao nível de atividade física, medido por meio do questionário de Baecke modificado, o grupo A obteve melhor média que o grupo $\mathrm{S}$, principalmente nas atividades esportivas e no total de horas praticadas, em que apresentou forte significância estatística, o que já era esperado por ser o grupo A composto de idosas ativas.

$\mathrm{Na}$ tabela 2 encontra-se este resultado, utilizado para selecionar as idosas ativas das sedentárias, separando-as em grupos.

Tabela 2 - Estatística descritiva e inferencial do questionário de Baecke entre os grupos de idosas. São José-SC, 2011.

\begin{tabular}{lccccccccc}
\hline & \multicolumn{2}{c}{ Média } & \multicolumn{2}{c}{ dp } & \multicolumn{2}{c}{ Mín. } & \multicolumn{2}{c}{ Máx. } & p-valor \\
\hline Atividades & A & S & A & S & A & S & A & S & \\
\hline Domésticas & 1,9 & 1,7 & 0,3 & 0,5 & 0,4 & 0,4 & 2,7 & 2,7 & 0,188 \\
Esportivas & 6,2 & 1,5 & 3,9 & 1,5 & 2 & 0 & 15,2 & 4 & $0,000^{*}$ \\
Lazer & 4,2 & 3,4 & 1,1 & 1,6 & 2,8 & 1,1 & 6,8 & 7,7 & 0,940 \\
Total horas & 12,1 & 6,7 & 4,1 & 2,3 & 7,3 & 2,4 & 20,1 & 9 & $0,000^{*}$ \\
\hline
\end{tabular}

$\mathrm{A}=\operatorname{ativas} ; \mathrm{S}=$ sedentárias; $\mathrm{dp}=$ desvio-padrão; Mín.= mínima; Máx.= máxima; $*$ significância - teste $t$ para amostras independentes.

Quanto aos resultados dos testes de aptidão física, ambos os grupos encontraram-se dentro do padrão de normalidade, segundo a classificação de Rikli \& Jones, ${ }^{17}$ exceto nos parâmetros "flexibilidade dos membros superiores" (alcançar atrás das costas com as mãos) e "resistência aeróbica" (andar seis minutos), que apresentaram desempenho inferior ao padrão para a idade.
Nos testes de flexibilidade de membros inferiores, mobilidade física - velocidade, agilidade e equilíbrio e resistência aeróbica, observou-se, entre os grupos, resultados com diferença estatisticamente significativa, como mostra a tabela 3 .

Tabela 3 - Comparação da aptidão física funcional entre os grupos de idosas (média \pm desvio-padrão). São José-SC, 2011

\begin{tabular}{lccc}
\hline & Grupo A & Grupo S & Valor de p \\
\hline $\begin{array}{l}\text { Força MMII } \\
\text { (repetições) }\end{array}$ & $12,0 \pm 4,3$ & $12,7 \pm 2,0$ & 0,365 \\
$\begin{array}{l}\text { Força MMSS } \\
\text { (repetições) }\end{array}$ & $15,5 \pm 2,9$ & $16,4 \pm 4,3$ & 0,262 \\
$\begin{array}{l}\text { Flexibilidade MI } \\
\text { (cm +/-) }\end{array}$ & $2,67 \pm 9,7$ & $-3,7 \pm 6,4$ & $0,031^{*}$ \\
$\begin{array}{l}\text { Flexibilidade MS } \\
\text { (cm +/-) }\end{array}$ & $-12,42 \pm 16,9$ & $-14,31 \pm 10,3$ & 0,365 \\
$\begin{array}{l}\text { Caminhada 2,44m } \\
\text { (segundos) }\end{array}$ & $6,08 \pm 0,7$ & $7,3 \pm 2,4$ & $0,049^{*}$ \\
$\begin{array}{l}\text { Caminhada 6min } \\
\text { (metros) }\end{array}$ & $451,8 \pm 49,8$ & $365,54 \pm 75,4$ & $0,001^{*}$ \\
\hline
\end{tabular}

Grupo A= idosas ativas (n=12); Grupo S= idosas sedentárias (n=13); * nível de significância 
Na avaliação da atenção por meio do TMT, encontraram-se valores médios de tempo de execução da parte A maiores que os valores de referência correspondentes para a faixa etária nos dois grupos (A e S). Na parte B, só o grupo A obteve o tempo de execução maior que os valores de referência correspondentes para a faixa etária, o que configura pior desempenho no teste, sendo que diferenças entre os grupos não foram estatisticamente significativas, como mostrado na tabela 4 .

Tabela 4 - Tempo de execução médio do Teste de Traços (TMT) das idosas. São José-SC, 2011

\begin{tabular}{|c|c|c|c|c|c|c|}
\hline & \multicolumn{3}{|c|}{ TMT parte A } & \multicolumn{3}{|c|}{ TMT parte B } \\
\hline & $\begin{array}{c}\text { Valor } \\
\text { encontrado }\end{array}$ & $\begin{array}{l}\text { Valor } \\
\text { de p }\end{array}$ & $\begin{array}{l}\text { Valor de } \\
\text { referência }\end{array}$ & $\begin{array}{c}\text { Valor } \\
\text { encontrado }\end{array}$ & $\begin{array}{l}\text { Valor } \\
\text { de p }\end{array}$ & $\begin{array}{l}\text { Valor de } \\
\text { referência }\end{array}$ \\
\hline & & & (Idade 60-85) & & & (Idade 60-85) \\
\hline \multirow{2}{*}{$\begin{array}{l}\text { Ativas } \\
\text { Sedentárias }\end{array}$} & $64,6( \pm 28,2)$ & \multirow{2}{*}{0,215} & $35,8( \pm 11,9)$ & $156,1( \pm 80,4)$ & \multirow{2}{*}{0,322} & $81,2( \pm 38,5)$ \\
\hline & $76,5( \pm 74,8)$ & & $60,7( \pm 26,0)$ & $147,0( \pm 83,0)$ & & $152,2( \pm 83,1)$ \\
\hline
\end{tabular}

Escores no Teste de Traços apresentados como segundos para completar cada uma das partes. Teste $t$ para amostras independentes.

Quando se analisou a distribuição quanto à categoria do TMT, observa-se que, na parte A deste teste, mais de $83 \%$ das idosas do grupo A ficaram abaixo dos valores de referência correspondentes para a faixa etária, enquanto que as do grupo $\mathrm{S}$ foram mais de $69 \%$. No TMT B, $75 \%$ do grupo A foram classificadas com o nível de atenção abaixo dos valores de referência, e do grupo S, 61,5\%.

\section{DISCUSSÃO}

A prática regular de exercícios físicos é reconhecida como uma forma de se prevenir e combater os males associados ao sedentarismo e a inatividade física, que quando combinada com o envelhecimento biológico pode resultar em perdas progressivas nas capacidades funcionais do indivíduo idoso. ${ }^{21}$

$\mathrm{Na}$ avaliação do nível de atividade física pela escala de Baecke, o presente estudo encontrou diferenças significativas nas atividades esportivas e no total de horas de atividades físicas semanais entre os dois grupos. Uma vez que o grupo A é composto de idosas ativas, tal fato é reforçado pelos achados de outro estudo, ${ }^{4}$ que concluiu que o programa de atividades físicas sistematizadas auxilia na manutenção da autonomia do idoso em relação às atividades da vida diária, e que os idosos percebem os benefícios proporcionados pela atividade física. No presente estudo, o grupo A realizava quase o dobro de atividades esportivas e quase o triplo de total de horas de atividades físicas semanais que o grupo $\mathrm{S}$, já que o mesmo é composto de idosas sedentárias.

As idosas apresentaram, de maneira geral, a aptidão física funcional preservada, o que pode ser justificado pela própria história de vida e pelas atividades cotidianas que realizam. Entretanto, sabe-se que o processo de perda funcional a partir dessa faixa etária é significativo e rápido. O início da prática de atividades físicas é recomendado para manter e minimizar as perdas funcionais, ${ }^{4,21}$ pois apesar da diminuição da aptidão física com o avanço da idade, essa alteração pode ser modificada com a prática de exercícios. ${ }^{22}$

Os resultados encontrados neste estudo demonstram que as idosas avaliadas atingiram bons níveis de aptidão física nos testes de força e resistência de membros superiores e inferiores, flexibilidade de membros inferiores e mobilidade física - velocidade, agilidade e equilíbrio. E 
níveis inferiores na flexibilidade dos membros superiores e resistência aeróbica em relação ao padrão de referência para a idade..$^{17}$

Em relação aos parâmetros da aptidão física funcional, flexibilidade de membros inferiores, mobilidade física e resistência aeróbica, o grupo das idosas ativas apresentou melhor desempenho, se comparadas com as idosas sedentárias. Este resultado corrobora pesquisas que relatam melhora da aptidão física funcional em idosos que praticam exercício físico..$^{4-7,21}$

A perda de força muscular é um processo natural que ocorre com o aumento da idade. As variáveis que tendem a apresentar padrões mais estáveis são a força dos músculos envolvidos nas atividades diárias, com contrações repetidas de baixa intensidade. Sofre maior declínio com a idade a força muscular dos músculos de atividades mais vigorosas, com contrações de velocidade rápida e produção de potência. ${ }^{23} \mathrm{O}$ fato de as idosas, em ambos os grupos, apresentarem boa manutenção da força dos membros superiores, pode ser associado à manutenção da atividade muscular durante as tarefas domésticas e de lazer.

A flexibilidade é uma das capacidades físicas que dependem do estado e condição das estruturas que envolvem as articulações. É considerada decisiva para o movimento, sendo um componente essencial da aptidão funcional do indivíduo, sobretudo para o idoso. Sua diminuição, além de reduzir a possibilidade de movimentar-se (andar, calçar um sapato, vestir um casaco), aumenta o risco de lesões nas articulações. ${ }^{24}$ Exercícios de flexibilidade não apenas aumentam a amplitude de movimento, mas também há evidências de que ocorrem diminuição de dores em geral, além de benefícios para as atividades de vida diária. ${ }^{25}$

O teste "alcançar atrás das costas" procura avaliar a movimentação geral do ombro: adução, abdução, rotação interna e externa. Candeloro \& Caromano $^{26}$ observaram melhoras significativas na mobilidade da cintura escapular e dos membros superiores em indivíduos idosos que participaram de um programa de exercícios regulares. Como o processo de deterioração osteoarticular se acelera a partir dos 65 anos, um pequeno aumento na amplitude de movimento advindo com trabalho de treinamento físico pode representar importante ganho na qualidade de vida dessas pessoas. Este fato contraria aos resultados encontrados neste estudo, no qual a perda da flexibilidade dos membros superiores ocorreu tanto nas idosas ativas quanto nas sedentárias.

Entretanto, é conhecido que ocorre queda da aptidão física com o envelhecimento, a qual se inicia de maneira gradativa, ao redor da quinta década de vida. Destacam-se os benefícios dos programas de exercícios físicos para idosos como medida profilática importante no sentido de preservar e retardar ao máximo os efeitos do envelhecimento sobre a aptidão física. ${ }^{27}$

$\mathrm{Na}$ análise da parte A do TMT, todas as idosas deste estudo apresentaram valores de tempo de execução maiores que os valores de referência correspondentes para a mesma faixa etária, o que demonstra um prejuízo na rapidez visomotora e na atenção. A atenção piora com o envelhecimento, o que pode estar associado à perda da independência funcional; ${ }^{28}$ entretanto, além desta perda previsível, observou-se no presente estudo, quando da análise da parte A do TMT, que todas as idosas apresentaram valores de tempo de execução maiores que os valores de referência correspondentes para a mesma faixa etária, o que demonstra prejuízo na rapidez visomotora e na atenção em ambos os grupos.

Verifica-se piora no desempenho em testes de função executiva com o avançar da idade, ${ }^{29}$ o que se apresentou apenas na amostra para o grupo A. Ao contrário do esperado, na execução da parte B do TMT, observou-se melhor desempenho do grupo S, já que somente o grupo A obteve tempo maior que os valores de referência correspondentes para a faixa etária, configurando pior função executiva deste grupo.

Ao final do estudo foi possível observar, apesar de quase todos os parâmetros da aptidão física das idosas estarem dentro dos padrões de normalidade, ${ }^{17}$ que esses parecem não garantir a 
manutenção de um bom funcionamento cognitivo. O baixo desempenho no teste de resistência aeróbica nos dois grupos talvez possa justificar o prejuízo encontrado na rapidez visomotora e na atenção de toda a amostra estudada.

Existe forte correlação entre o aumento da capacidade aeróbica e a melhora em funções cognitivas. As perdas cognitivas estão relacionadas à redução da função cardiovascular decorrente do envelhecimento, o que levaria a um decréscimo progressivo na oxigenação e uma hipóxia tecidual ao longo do tempo, implicando, por fim, declínio cognitivo. Acredita-se que a prática regular do exercício físico poderia influenciar positivamente nessa situação, diminuindo ou retardando o ritmo deste processo involutivo. ${ }^{30}$ Destaca-se ainda a importância de praticar exercícios que estimulem as capacidades intelectuais, além das capacidades físicas nesses idosos.

As limitações deste estudo estão relacionadas ao número reduzido da amostra, pois algumas idosas não se dispuseram a participar do estudo, além de algumas terem desistido durante sua

\section{REFERÊNCIAS}

1. Salin MS, Mazo GZ, Cardoso AS, Garcia GS. Atividade Física para idosos: diretrizes para implantação de programas e ações. Rev Bras Geriatr Gerontol 2011;14(2):197-208.

2. Matsudo SM. Envelhecimento e atividade física. Londrina: Midiograf; 2001. 195 p.

3. Blair SN. Physical Inactivity: the major public health problem of the new millennium the evidence and strategies for change. In: Anais do Congresso Desporto, Actividade Física e Saúde: o contributo da ciência e o papel da escola. Porto, Portugal: FCDEF; 2002. p. 2-4.

4. Kuwano VG, Silveira AM. A influência da atividade física sistematizada na autopercepção do idoso em relação às atividades da vida diária. Revista da Educação Física 2002;13(2):35-40.

5. Landi F, Onder G, Carpenter I, Cesari M, Soldato M, Bernabei R. Physical activity prevented functional decline among frail community-living elderly subjects in an international observational study. J Clin Epidemiol 2007;60(5):518-24. realização, por não concluírem os testes de aptidão física. Outra potencial limitação são os estudos reduzidos com a mesma amostra e variáveis, o que impossibilita a comparação dos resultados.

\section{CONCLUSÃO}

Neste estudo, foi possível observar que a aptidão física funcional das idosas fisicamente ativas e sedentárias está dentro dos padrões de normalidade, com bons níveis de aptidão física nos testes de força e resistência de membros superiores e inferiores, flexibilidade de membros inferiores e mobilidade física - velocidade, agilidade e equilíbrio. Os níveis são inferiores na flexibilidade dos membros superiores e resistência aeróbica em relação ao padrão de referência para a idade. As idosas ativas apresentaram melhor flexibilidade de membros inferiores, mobilidade física e resistência aeróbica. Nos dois grupos, constatou-se prejuízo na atenção e na rapidez visomotora. Destacase a importância de se praticar exercícios que estimulem as capacidades intelectuais, além das capacidades físicas dos idosos.

6. Paes JO, Duarte YAO, Lebrão ML, Santos JLF, Laurenti R. Impacto do sedentarismo na incidência de doenças crônicas e incapacidades e na ocorrência de óbitos entre os idosos do município de São Paulo. Saúde Coletiva 2008;5(24):183-8.

7. Wilmore JH, Costill DL. Fisiologia do esporte e do exercício. São Paulo: Manole; 2001. 709 p.

8. Costa AV. A atenção e velocidade perceptiva na mulher idosa: um estudo com praticantes e não praticantes de atividade desportiva. [dissertação de Mestrado]. Porto: Faculdade de Desporto da Universidade do Porto; 2007.

9. Cardoso AS, Japiassú AT, Cardoso LS, Levandoski G. O processo de envelhecimento do sistema nervoso e possíveis influências da atividade física. Publ UEPG Ci Biol Saúde 2007;13(3/4):29-44.

10. McAuley E, Kramer AF, Colcombe SJ. Cardiovascular fitness and neurocognitive function in older adults: a brief review. Brain Behav Immun 2004;18(3):214-20. 
11. Kramer AF, Colcombe SJ, McAuley E, Scalf PE, Erickson KI. Fitness, aging and neurocognitive function. Neurobiol Aging 2005; 26(Suppl 1): 124-7.

12. Miranda LM, Farias SF. A influência do exercício físico na atividade cerebral do idoso. Rev EFDeportes [periódico na internet]. 2008 [acesso em 5 set 2011];13(125):[aproximadamente 5 p.]. Disponível em: http://www.efdeportes.com/efd125/a-influencia-doexercicio-fisico-na-atividade-cerebral-do-idoso.htm

13. Alencar NA, Souza Júnior JV, Aragão JCB, Ferreira MA, Dantas E. Nível de atividade física, autonomia funcional e qualidade de vida em idosas ativas e sedentárias. Fisioter Mov 2010;23(3):473-81.

14. Mazo GZ, Gioda FR, Schwertner DS, Galli VLB, Guimarães ACA, Simas JPN. Tendência a estados depressivos em idosos praticantes de atividade física. Rev Bras Cine Des Hum 2005;7(1):45-9.

15. Mazo GZ, Mota J, Benedetti TB, Barros MVG. Validade concorrente e reprodutibilidade: testereteste do questionário de Baecke modificado para idosos. Rev Bras Ativ Fís Saúde 2001;6(1):5-11.

16. Voorrips LE, Ravelli AC, Dongelmans PC, Deurenberg P, Van Staveren WA. A physical activity questionnaire for the elderly. Med Sci Sports Exerc 1991;23(8):974-9.

17. Rikli RE, Jones CJ. Senior fitness test manual. Champaign, Illinois: Human Kinetics: Human Kinetics; 2001. 176 p.

18. Bertolucci PHF, Brucki SMD, Campacci SR, Juliano Y. O mini-exame do estado mental em uma população geral: impacto da escolaridade. Arq Neuropsiquiatr 1994;52(1):1-7.

19. Strauss E, Sherman EMS, Spreen O. A compendium of neuropsychological tests: administration, norms and commentary. New York: Oxford University Press; 2006; 1240 p.

20. Tombaugh TN. Trail Making Test A and B: normative data stratified by age and education. Arch Clin Neuropsychol 2004;19(2):203-14.
21. Rogatto GP, Gobbi S. Efeitos da atividade física regular sobre parâmetros antropométricos e funcionais de mulheres jovens e idosas. Rev Bras Cineantropom Desempenho Hum 2001;3(1):63-9.

22. Alves RV, Mota J, Costa MC, Alves JGB. Aptidão física relacionada à saúde de idosos: influência da hidroginástica. Rev Bras Med Esporte 2004;10(1):31-7.

23. Freitas EV, Py L, Néri AL, Cançado FAX, Gorzoni ML, Rocha SM, editores. Tratado de Geriatria e Gerontologia. Rio de Janeiro: Guanabara Koogan; 2002.

24. Cunha RCL, Araújo JP, Aragão JCB, Dantas EHM. Análise da flexibilidade e da autonomia funcional em idosos não praticantes de exercício físico. In: Livro de Memórias do III Congresso Científico Nortenordeste. Fortaleza: CONAFF; 2007; p. 162-8.

25. Nunes MES, Santos S. Avaliação funcional de idosos em três programas de atividade física: caminhada, hidroginástica e Lian Gong. Rev Port Cien Desp 2009;9(2-3):150-9.

26. Candeloro JM, Caromano FA. Efeito de um programa de hidroterapia na flexibilidade e na força muscular de idosas. Rev Bras Fisioter 2007;11(4):303-9.

27. Pereira FD, Batista WO, Furtado HL, Alves Junior ED, Giani TS, Dantas EHM. Comparação da força funcional de membros inferiores e superiores entre idosas fisicamente ativas e sedentárias. Rev Bras Geriatr Gerontol 2009;12(3):417-27.

28. Custódio EB, Malaquias Júnior J, Voos MC. Relação entre cognição (função executiva e percepção espacial) e equilíbrio de idosos de baixa escolaridade. Fisioter Pesqui 2010;17(1):46-51.

29. Ashendorf L, Jefferson AL, O'Connor MK, Chaisson C, Green RC, Stern RA. Trail Making Test errors in normal aging, mild cognitive impairment, and dementia. Arch Clin Neuropsychol 2008;23(2):129-37.

30. Ble A, Volpato S, Zuliani G, Guralnik JM, Bandinelli $S$, Lauretani F, et al. Executive function correlates with walking speed in older persons: the InCHIANTI study. J Am Geriatr Soc 2005;53(3):410-5. 